\title{
Potential occupational and environmental factors in SSc onset
}

\author{
Irena Walecka', Marek Roszkiewicz' , Aleksandra Malewska' \\ Dermatology Department, Central Clinical Hospital of the Ministry of the Interior and Administration, Warsaw, Poland \\ Walecka I, Roszkiewicz M, Malewska A. Potential occupational and environmental factors in SSc onset. Ann Agric Environ Med. 2018; 25(4): \\ 596-601.
}

\begin{abstract}
Introduction and objective. Systemic sclerosis [SSc; scleroderma] is a rare, connective tissue disorder affecting all organs and systems. The primary feature of this disease is a chronic, progressive fibrosis due to excessive production of collagen and other components. There are two subsets of systemic sclerosis: 1) diffused SSc (dSSc), 2) limited SSc (ISSc) and 3) scleroderma without sclerosis (SSSC). The presented review is an attempt to summarize recent data regarding environmental and occupational factors in SSc onset.

State of knowledge. There are many factors to be taken under consideration with SSc onset, although a strong correlation has been established for only a few. The most distinct factors are: crystalline silica and organic solvents (such as white spirit, aromatic, aliphatic-chain, chlorinated solvents, ketones, welding fumes). For other factors, which include abstestos, air pollution, other chemicals, silicone breast implants, tobacco smoking, drug reactions, diet influence and exposure to heavy metals, the jury is still out, and their position in SSc onset needs further studies.

Conclusions. Although the pathogenesis of scleroderma remains unclear, there is a marked correlation between the onset of SSc and certain environmental or occupational factors.
\end{abstract}

\section{Key words}

systemic sclerosis, connective tissue disease, environmental factors, occupational exposure, silica

\section{INTRODUCTION}

Systemic sclerosis [SSc; scleroderma] is a rare, chronic, inflammatory connective tissue disease which affects all organs and systems [1]. The most prominent feature of this condition is a diffused progressive fibrosis due to excessive deposition of extracellular matrix components in various tissues $[1,2]$. Systemic sclerosis is also characterized by generalized microangiopathy, activation of the immune system, and production of different kinds of autoantibodies $[1,3]$.

According to the latest classification, there are three main types of scleroderma: diffused scleroderma (dSSc), limited scleroderma (1SSc) and scleroderma without sclerosis (SSc sine sclerodema, SSSC) [4]. In its limited form, systemic sclerosis skin changes are confined to the acral parts of the body (hands, feet, face). Raynaud's phenomenon is observed many years before diagnosis and the course of the illness is relatively slow and mild with good general prognosis (a ten-year survival rate in up to $90 \%$ of cases). Organ involvement in ISSc is observed in late-stage disease, contrary to $[3,5] \mathrm{dSSc}$ where organ involvement is one of the first signs of the disease. Patients with the diffused form of SSc have advanced generalized skin fibrosis, and the onset of Raynaud's phenomenon is observed, on average, one year before skin lesions appear. The general prognosis for patients with dSSc is poor, with a ten-year survival rate in up to $74 \%$ of cases [5, 6]. SSSC is a rare form of the disease in which vascular and fibrotic damage to the internal organs is present (similar to ISSc), but without cutaneous sclerosis $[4,7]$.

Clinical presentation of this disease is extremely heterogeneous and depends on the degree of organ

Address for correspondence: Irena Walecka, Dermatology Department, Central Clinical Hospital of the Ministry of the Interior and Administration in Warsaw, Poland e-mail: irena.walecka@cskmswia.pl

Received: 22 February 2017; accepted: 19 May 2017; first published on July 2017 involvement [1]. Systemic sclerosis may affect multiple internal organs, such as the kidneys, lungs, heart and the gastrointestinal tract, leading to their failure.

The main dysfunctions are vascular changes leading to peripheral microcirculation disturbance, which manifest as skin lesions typical of systemic sclerosis, and include Raynaud's phenomenon [Fig. 1], skin fibrosis, microstomia [Fig. 2], ulceration of the distal phalanges [Fig. 3], diffused soft tissue calcification [Fig. 4] and oedema of the fingers [8,9].

Raynaud's phenomenon is one of the most characteristic symptoms of systemic sclerosis, resulting from a microvascular

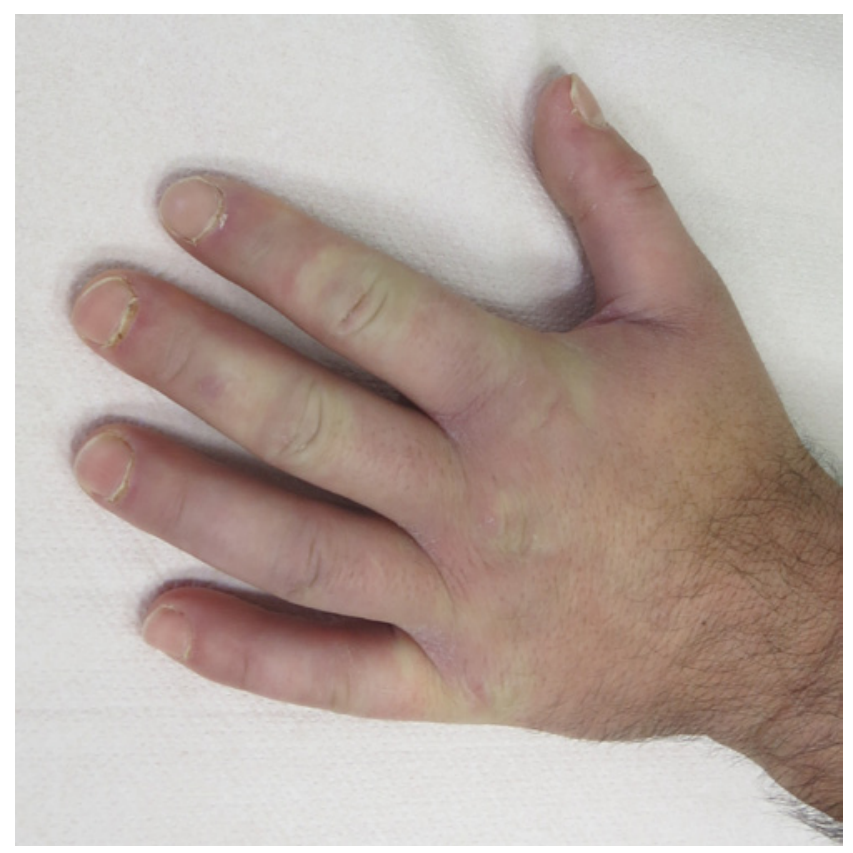

Figure 1. Raynaud's phenomenon 


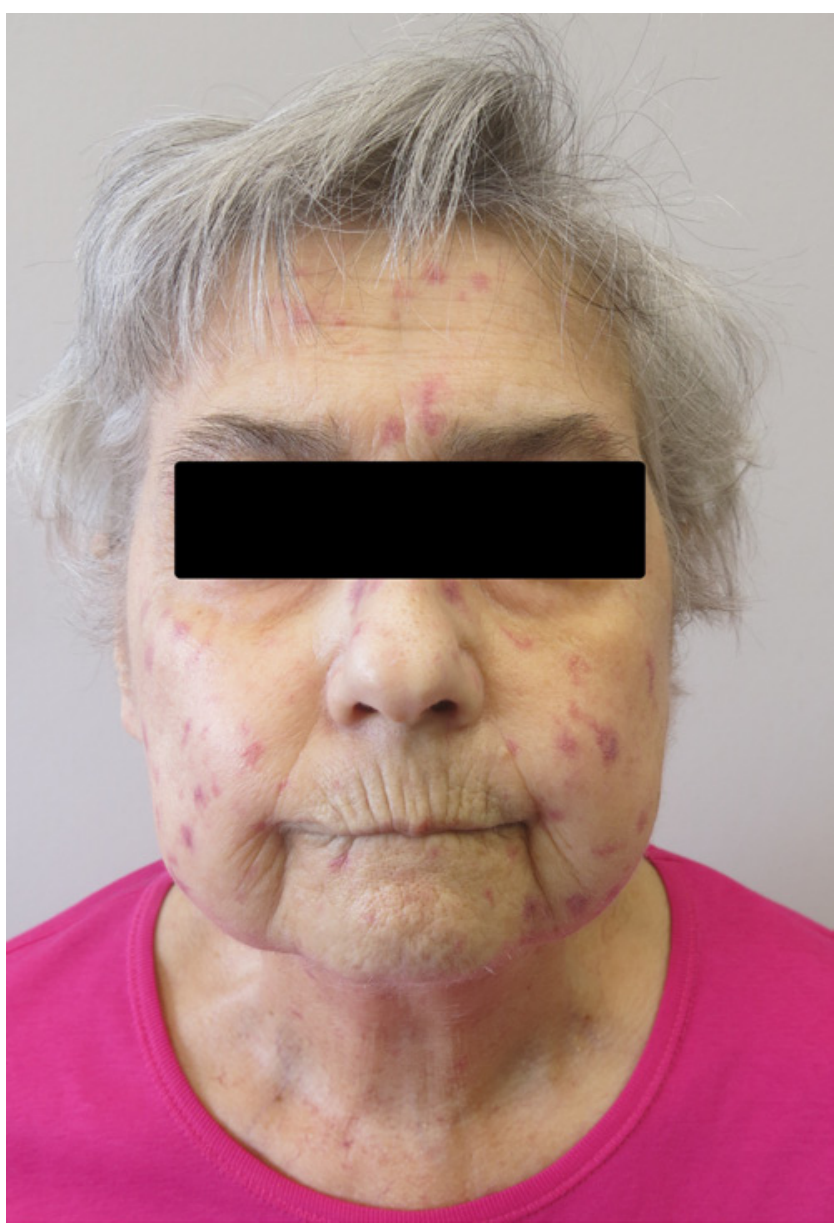

Figure 2. Microstomia

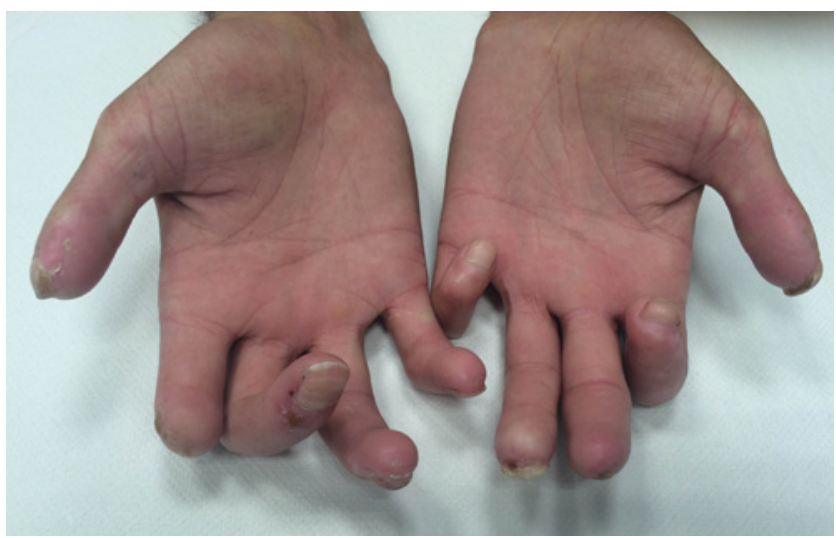

Figure 3. Digital acroosteolysis, amputation of distal phalange, erosions

function disorder where a recurrent, reversible vasospasm of distal small vessels is observed. This phenomenon mainly affects vessels such as small arteries (arterioles), precapillary vessels, as well as postcapillary vessels [10]. Exposure to cold is the strongest factor, which triggers Raynaud's phenomenon.

Involvement of the lungs causes interstitial lung disease (ILD) - pulmonary fibrosis and pulmonary arteries leading to hypertension - and is the most severe condition. ILD manifests in a lower lung function test (lower FVC, TVC) and imaging (honeycombing, beaded bronchiectasis) [Fig. 5, 6]. Morbidity under those conditions is significantly higher than in other

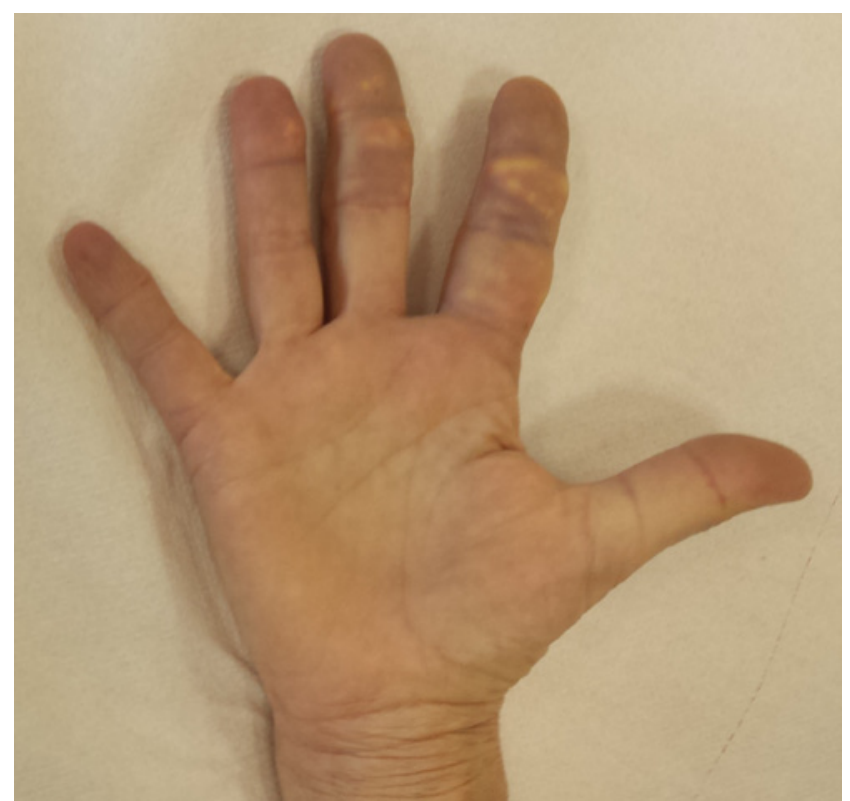

Figure 4. Soft tissue calcification

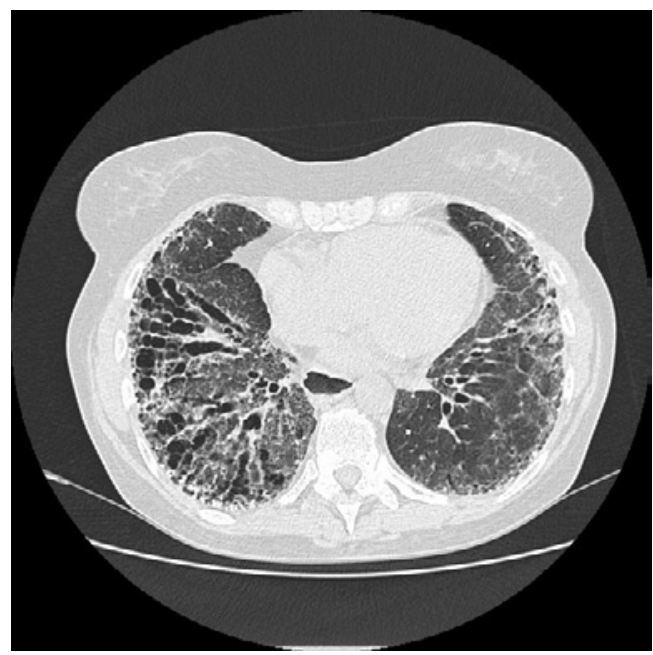

Figure 5. Honeycomb changes in both lungs in ILD

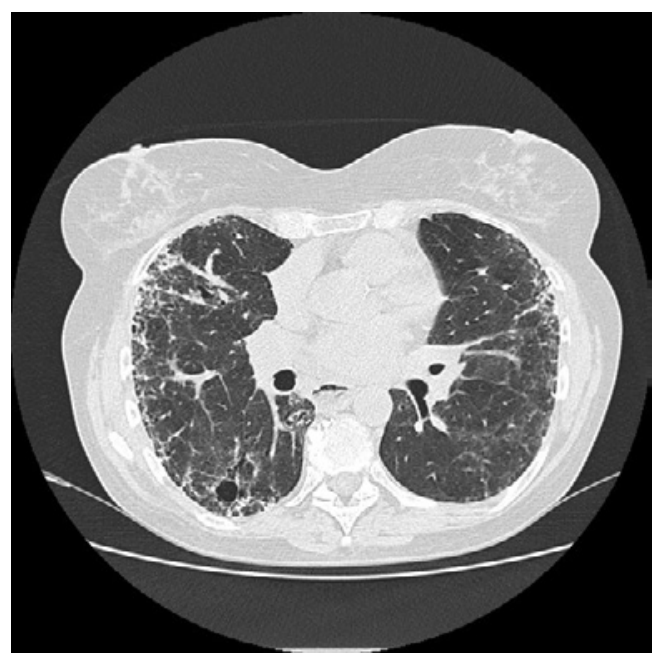

Figure 6. Multiple beaded bronchiectasis in both lungs 
patient groups (ILD - 35\%, PAH - 26\%) [11]. This is why proper diagnostics and regular lung function checks must be carried out. If untreated, SSc-related PAH (SSc-PAH) a rapidly fatal course may follow, with death resulting from right ventricular failure and arrhythmias [11].

Scleroderma symptoms also manifest in the cardiovascular system, which is particularly difficult to diagnose, because it often remains subclinical. However, up to $35 \%$ of patients with scleroderma (both diffused SSc and limited SSc) suffer from cardiovascular problems [12]. Scleroderma can affect every structure of the heart: pericardium, myocardium and pericardium, as well as the electrical conduction system. This is why the spectrum of symptoms is very wide and diverse. It may cause fibrinous pericarditis, myocardial dysfunction resulting in ventricular insufficiency, multiple valvular dysfunctions and arrhythmias [13]. The biggest challenge is that due to the lack of distinct symptoms, the great majority of patients remain undiagnosed. Without proper treatment in the early stages of the disease, complications progress silently. Unfortunately, once the heart-related problems becomes symptomatic, the prognosis is very poor [14].

During the course of SSc, kidney function is also impaired. A decreased glomerular filtration rate (GFR) is common in SSc. Most patients have mild or moderate renal insufficiency [15], although some patients have end-stage renal disease necessitating haemodialysis therapy and transplantation. Kidney transplantation is one of the therapeutic options for end-stage renal disease (ESRD) in systemic sclerosis (SS), but current evidence suggests poorer patient and graft survival after transplantation in SS than in the case of other primary kidney diseases. Previously diagnosed arterial hypertension, especially when treated with ACE inhibitors or diuretics and glucocorticoids, are independent risk factors associated with a decreased GFR in SSc [15].

The gastrointestinal tract (GIT) is a very common site of visceral manifestations of scleroderma - about $90 \%$ of patients suffer from GIT symptoms. Gastrointestinal involvement is a very common cause of morbidity among such patients, in addition to cardiovascular, pulmonary and renal complications [16]. The most common site of GIT involvement is the oesophagus. Scleroderma leads to smooth muscle atrophy and fibrosis of the distal part of the oesophagus, resulting in abnormal peristalsis. Patients often suffer from gastroesophageal reflux disease, heartburn and regurgitations [17]. Scleroderma may also be the cause of pseudoobstruction, malabsorption syndrome and motility disorders. Although most GIT manifestations are not lifethreatening, they profoundly influence the quality of life of scleroderma patients [18].

In SSc diagnostics, as well as in other connective tissue diseases, blood serum tests for autoantibodies and nailfold videocapilalroscopy are important (NVC), in addition to specific organ tests (ultrasonography, computed tomography etc.).

The antibodies associated with $\mathrm{SSc}$ are antinuclear (ANA/ENA) and anticentromere (ACA). Among different ENA the most common subtypes are anti-topoisomerase I (Scl-70/TOPO I). An increasing number of studies indicate an association between different kinds of antibodies and organ involvement. A distinct connection has been established between ACA (37\%), Scl-70 (24\%) and cardiopulmonary involvement [19]. Anti-RNAP3+ patients with SSc have a high risk of concomitant malignancy and regular screening for cancer is recommended [20]. Scleroderma patients who are double-positive for antibodies recognizing ACA and antiinterferon inducible protein 16 (IFI-16), are significantly more likely to experience significant digital vascular events [21]. Regardless of the ENA-subtype, nailfold videocapillaroscopy pattern showed a stable association with a presence of interstitial lung disease (ILD) or elevated systolic pulmonary artery pressure (sPAP $>35 \mathrm{mmHg}$ ) [19].

A further significantly important test in SSc diagnostics is NVC. This non-invasive method uses a magnifying technique in order to evaluate the morphology of the capillaries of the nail bed, where the vessels are most visible owing to their parallel course against the skin surface [22]. It has been established that changes in NVC can be present in patients with Raynaud's phenomenon, even without any other clinical presentation of SSc for several years. In such cases, a characteristic scleroderma pattern is described. There are three stages: early, active and late. They are characterized by different evaluation of a few elements such as: enlarged/ gigantic vessels (loops), distribution of the vessels, presence of haemorrhages or oedema, avascular regions and multiple branched capillaries [Fig. 7] [22].

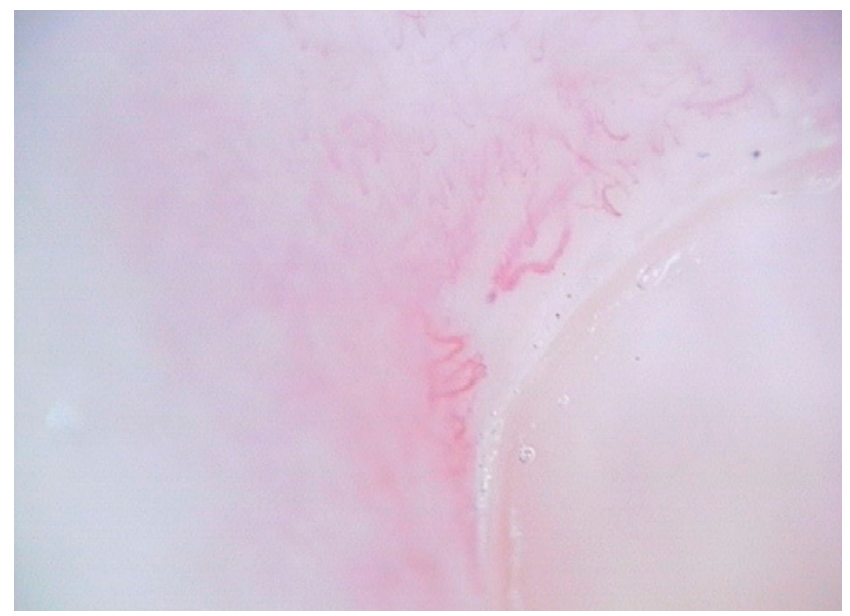

Figure 7. Irregular nailfold's capilars in NVC

The pathogenesis of systemic scleroderma is based on three distinct phenomena: diffused microangiopathy, a production of autoantibodies (triggering chronic inflammation) and excessive production of ECM components, such as collagene, due to fibroblast disfunction $[2,9,23]$.

Many facts remain unclear in the field of systemic scleroderma pathogenesis; however, research suggests a certain correlation between the pathogenesis and the environment. There are multiple publications supporting the hypothesis that environmental factors are crucial in the development of scleroderma [2].

There are ongoing studies into the establishment of specific genes associated with SSc, and even particular genes connected with organ involvement [24-26]. It is clear, however, that gene prevalence is not the only a determinant as to whether a person will develop this kind of disease or not, there are also other factors that can play a role. There are numerous studies on the environmental and occupational factors associated with SSc onset. Sometimes, the factor is relatively easy to establish, but in a majority of cases, such an association, remains unclear or a cumulative influence must be taken into consideration. 
The presented review is an attempt to summarize the already known factors playing a role in SSc onset (such as silica and organic solvents), and recent data concerning other environmental and occupational factors (air pollution, asbestos, drugs, breast implants, dyes and heavy metals).

State of knowledge - Silica. The pathogenic mechanisms of crystalline silica in the development of SSc remain unclear [27]. Silica is known to be a T cell adjuvant. Activated T cells (mainly Th17 and Th1) start to trigger the inflammatory process (higher concentration of pro-inflammatory cytokines, such as: tumour necrosis factor TNF, interferon gamma, interleukin 17A in bronchoalveolar lavage fluid in experimental models), which causes the development of fibrosis due to the activation of fibroblasts $[28,29]$.

The first report of the onset of scleroderma in connection with occupational factors was provided by Bramwell in 1914, regarding two Scottish stonemasons [30]. In 1957, Erasmus also reported a higher number of scleroderma patients in 17 gold miners working with silica dust [31]. It was evaluated that patients exposed to silica dust have a 25 times higher risk of scleroderma, and an even 110 times higher risk with frank silicosis [32]. In 2014, a case-controlled study showed a strong association between systemic sclerosis and silica dust exposure, as well as a higher risk in the male population, and was associated with a higher cumulative exposure score [33].

Silica exposure has also been linked to SSc severity (a higher Rodnan's scale score, digital ulcers, interstitial lung disease and lower vital parameters, such as forced vital capacity, vital capacity, left ventricular ejection volume, and a diffusing of lung capacity for carbon monoxide) [33]. Survival rates are lower in SSc patients exposed to silica than in those with its 'idiopathic' form [34]. The majority of patients exposed to silica dust were foundry or construction workers and roofers [33].

Organic solvents and other chemicals. There are numerous reports on the association of scleroderma with organic solvents, including different compounds, i.e. white spirit, aromatic, aliphatic-chain, chlorinated solvents, ketones and welding fumes) [27, 35-37]. These substances are commonly used in dry cleaning, welding, painting, varnishing, glue solvents, nail-polish removers and industry (petrol, pharmaceuticals, epoxy resin, and vinyl chloride manufacturing). Workers in such industries and other similar industries are particularly at risk.

In May 1981, there was an epidemic of a previously unrecognized multisystem scleroderma-like disease in Spain [38]. It was been caused by rapeseed cooking oil polluted by aniline.

Other factors - Air pollution. Particulate emissions from diesel engine exhausts has been associated with fibrosis [1]. Diesel nanoparticles stimulate pro-inflamatory and profibotic processes in human skin causing fibrosis. However, a formal link between air pollution and SSc onset is yet to be proved [39].

Asbestos. To-date, asbestos exposure has not been strongly linked with any particular autoimmune or connective tissue disorder [40]. Although Noonan et al. in 2006 showed an association between asbestos exposure and scleroderma, the study population was relatively small and such a frequency was to be expected [40]. Nevertheless, Gold et al. reported an increased risk of death in patients with scleroderma and previous exposure to asbestos. Further study is still required before any firm conclusions can be reached.

Smoking and drugs. Cigarette smoking is known to have a negative effect on vascular and pulmonary outcomes in scleroderma patients. However, there is no evidence confirming SSc onset due to smoking itself.

Certain drugs are considered to be associated with the induction of sclerosis, such as bleomycin or paklitaksel [41]. Cocaine has been linked to scleroderma-like syndromes [41, 42], whereas a recent review paper has shown that there is no firm evidence of a correlation between drugs (anorexigens, pentazocine, bromocriptine, I-tryptophan) and SSc onset [27]. The authors indicate that their data and recent literature review are insufficient to establish a causative link between drugs and SSc onset [27].

Silicone. A report by Maria I et al. established that there is no correlation between silicone breast implants (SBI) and SSc onset [27]. However, Saiqusa et al. have recently investigated the association of SBI history with the development of SSc positive for anti-RNAP III antibody [43]. Among Japanese patients there was a higher number of women with a history of silicone breast implants and anti-RNAP III antibody, compared with the anti-TOPO I and the ACA group. The research findings remain very inconclusive.

Heavy metals. As in other autoimmune diseases, such as rheumatoid arthritis or systemic lupus erythematosus, a link with occupational exposure to heavy metals has been established $[44,45]$. A recent case-controlled study by Maria I et al., using a hair sample analysis, investigated the risk of SSc in patients exposed to heavy metals [46]. The concentrations of different heavy metals are usually 10 times higher in hair than in urine or blood samples. In the study, 100 patients with SSc and 300 controls were enrolled. Unlike the SSc patients, all controls came from the same region, i.e. Normandy in France. The study showed that there is a correlation between SSc and exposure to antimony, cadmium, lead, mercury, molybdenum, palladium and zinc [46].

Diet. Although there are no studies that show a direct influence of diet and food on SSc onset, a recent small case-controlled study (19 SSc patients and 20 controls) by Hughes M. et al. assessed that the selenium concentration of a SSc patient's serum was significantly lower than that of the controls [47]. During the last few years, the role of selenium as an antioxidant has been emphasized in the orbital fibrosis process in Graves-Basedow disease [48]. Selenium abolishes some of the effects of oxidative stress in orbital fibroblasts, namely, increased proliferation and secretion of pro-inflammatory cytokines [48]. If reduced selenium levels contribute to oxidative stress (implicated in the pathogenesis of SSc) and to fibrosis, then supplementation could represent a simple therapeutic target for intervention [47]. Perhaps studies regarding selenium concentration in soil and water and SSc onset, or in the course of the disease, may demonstrate the role of selenium deficiency in profibrotic and oxidative stress processes, as in the studies by Aleksandrowicz J. et al. showing, for example, the role of 
magnesium deficiency in leukaemia development among the population of the Kraków region in Poland [49-53].

\section{CONCLUSIONS}

There are numerous studies that suggest a relationship between SSc and environmental and occupational factors. A marked correlation of SSc onset was established in occupational exposure to different agents: crystalline silica dust and organic solvents (such as white spirit, ketones, aromatic, aliphatic-chain, chlorinated solvents, welding fumes); however, as yet, there is insufficient data to establish further correlation. Asbestos and air pollution (diesel engine exhausts) are factors that may play a role in SSc onset. Other factors (e.g. silicone breast implants, exposure to heavy metals, selenium deficit) also need to be further investigated.

It has been shown that the severity of SSc and SSc outcomes are strongly connected with environmental factors. Exposed patients tend to develop more severe forms of SSc (higher Rodnan's scale score, larger number of digital ulcers, ILD, poorer survival outcomes), and it would therefore be of great clinical value to establish the potential occupational or environmental factors more precisely.

\section{REFERENCES}

1. Almeida C, Almeida I, Vasconceloset C. Quality of life in systemic sclerosis. Autoimmunity Rev. 2015; 14: 1087-1096.

2. Barsotti S, Stagnaro C, d'Ascanio A, Della Rossa A. One year in review 2016: systemic sclerosis. Clin Exp Rheumatol. 2016; 34 Suppl 100(5): $3-13$.

3. Kowal-Bielecka O. Twardzina układowa - aktualności. Annales Academiae Medicae Stetinesis 2010; 56, suppl. 1: 80-82.

4. Szymańska E, Wieczorek M, Łagun Z, et al. Vascular changes in autoimmunological connective tissue diseases. Acta Angiol Vol. 22, No. 4 pp. 1-4 doi: 10.5603/AA.2016.0001

5. Kowal-Bielecka O, Kuryliszyn-Moskal A. Twardzina układowa. Reumatologia 2012; 50.2: 124-129.

6. LeRoy EC, Black C, Fleischmajer R. SSc, Diffuse Cutaneous. "Scleroderma (systemic sclerosis): classification, subsets and pathogenesis." J Rheumatol. 1988; 15: 23.

7. Szymańska E, Maj M, Rudnicka L. Twardzina układowa - przebieg kliniczny i możliwości terapeutyczne. Przegl Lek.2005; 62: 1538-1541.

8. Masi AT. Preliminary criteria for the classification of systemic sclerosis (scleroderma). Arthritis Rheumatol. 1980; 23.5: 581-590.

9. Anne Claire Desbois AC, Cacoub P. Systemic sclerosis: An update in 2016. Autoimmunity Rev. 2016; 15: 417-426.

10. Bonnecaze, Alex K. Raynaud's phenomenon in limited cutaneous systemic sclerosis. BMJ case reports 2015 (2015).

11. Tyndall AJ, Bannert B, Vonk M, et al. Causes and risk factors for death in systemic sclerosis: a study from the EULAR Scleroderma Trials and Research (EUSTAR) database. Ann Rheum Dis. 2010 Oct; 69(10): 1809-15. doi: 10.1136/ard.2009.114264.

12. Kahan A, Coghlan G, McLaughlin V. Cardiac complications of systemic sclerosis. Rheumatology (Oxford) 2009; 48.Suppl 3: iii45-iii48.

13. Champion HC. The heart in scleroderma. Rheum Dis Clin North Am. 2008; 34(1): 181-190.

14. Marasini B, Massarotti M, Cossutta R. Scleroderma heart disease. Int J Immunopathol Pharmacol. 2005; 18.4: 609-614.

15. Ostojic $\mathrm{P}$, Stojanovski N. Arterial hypertension treated with angiotensin converting enzyme inhibitors and glucocorticoids are independent risk factors associated with decreased glomerular filtration rate in systemic sclerosis. Rheumatol Int. 2016 Dec 22. doi: 10.1007/s00296-016-3632-y.

16. Thoua Nora M, et al. Assessment of gastrointestinal symptoms in patients with systemic sclerosis in a UK tertiary referral centre. Rheumatology 2010; 49.9: 1770-1775.

17. Ebert, Ellen C. Esophageal disease in scleroderma. J Clin Gastroenterol. 2006; 40.9: 769-775.
18. Rose, Suzanne, Michele A. Young, and James C. Reynolds. Gastrointestinal manifestations of scleroderma. Gastroenterology Clinics of North America 1998; 27.3: 563-594.

19. Markusse IM, Meijs J, de Boer B et al. Predicting cardiopulmonary involvement in patients with systemic sclerosis: complementary value of nailfold videocapillaroscopy patterns and disease-specific autoantibodies. Rheumatology (Oxford). 2016 Dec 10. pii: kew402.

20. Lazzaroni MG, Cavazzana I, Colombo E, et al. Malignancies in Patients with Anti-RNA Polymerase III Antibodies and Systemic Sclerosis: Analysis of the EULAR Scleroderma Trials and Research Cohort and Possible Recommendations for Screening. J Rheumatol. 2017 Jan 15. pii: jrheum.160817. doi: 10.3899/jrheum.160817.

21. McMahan ZH, Wigley FM, Casciola-Rosen L. Increased risk of digital vascular events in scleroderma patients who have both anti-centromere and anti-interferon-inducible protein 16 antibodies. Arthritis Care Res (Hoboken). 2016 Jul 7. doi: 10.1002/acr.22978.

22. Walecka I, Malewska A, Roszkiewicz M et al. Raynaud's phenomenon - the clinical picture, treatment and diagnostics. Acta Angiol. 2016; 22(4): 1-4 doi: 10.5603/AA.2016.0001.

23. Wollheim FA. Classification of systemic sclerosis: visions and reality. Rheumatology (Oxford). 2005; 44: 1212-6.

24. Chairta P, Nicolaou P, Christodoulou K. Genomic and genetic studies of systemic sclerosis: A systematic review. Hum Immunol. 2017 Feb; 78(2): 153-165. doi: 10.1016/j.humimm.2016.10.017.

25. Chen JQ, Papp G, Szodoray P, et al. The role of microRNAs in the pathogenesis of autoimmune diseases. Autoimmun Rev. 2016 Dec; 15(12): 1171-1180. doi: 10.1016/j.autrev.2016.09.003.

26. Carmona FD, Onat AM, Fernández-Aranguren T. Analysis of Systemic Sclerosis-associated Genes in a Turkish Population. J Rheumatol. 2016 Jul; 43(7): 1376-9. doi: 10.3899/jrheum.160045.

27. Marie I, Gehanno JF. Environmental risk factors of systemic sclerosis. Semin Immunopathol. 2015 Sep; 37(5): 463-73.

28. Liu T, Dai W, Li C, et al. Baicalin Alleviates Silica-Induced Lung Inflammation and Fibrosis by Inhibiting the Th17 Response in C57BL/6 Mice. J Nat Prod. 2015 Dec 24; 78(12): 3049-57.

29. Li C, Du S, Lu Y et al. Blocking the 4-1BB Pathway Ameliorates Crystalline Silica-induced Lung Inflammation and Fibrosis in Mice. Theranostics. 2016 Sep 9; 6(12): 2052-2067. eCollection 2016.

30. Bramwell B. Diffuse scleroderma: its frequency, its occurrence in stone masons, its treatment by fibrolysinelevations of temperature due to fibrolysin injections. Edinburgh MedJd 1914; 12: 387-401.

31. Erasmus L D. Scleroderma in gold miners on the Witz Witwatersrand with particular reference to pulmonary manifestations. S Afry Lab Clin Med. 1957; 3: 209-31.

32. Haustein U F, Ziegler V. Environmentally induced systemic sclerosislike disorders. Int3J Dermatol. 1985; 24: 147-51.

33. Marie I, Gehanno JF, Bubenheim M, et al. Prospective study to evaluate the association between systemic sclerosis and occupational exposure and review of the literature. Autoimmun Rev. 2014 Feb; 13(2): 151-6.

34. Freire M, Alonso M, Rivera A et al. Clinical peculiarities of patients with scleroderma exposed to silica: A systematic review of the literature. Semin Arthritis Rheum. 2015 Dec; 45(3): 294-300. doi: 10.1016/j. semarthrit.2015.06.004.

35. Magnant J, de Monte M, Guilmot JL, et al. Relationship between occupational risk factors and severity markers of systemic sclerosis. J Rheumatol. 2005; 32: 1713-1718.

36. Walder BK. Solvents and scleroderma. Lancet 1965; ii: 436-7.

37. Czirjak L, Katalin D, Schlammadinger J et al. Progressive systemic sclerosis occurring in patients exposed to chemicals. IntJ Dermatol 1987; 26: 374 .

38. Tabuenca JM. Toxic-allergic syndrome caused by ingestion of rapeseed oil denatured with aniline. Lancet. 1981 Sep 12;2(8246): 567-8.

39. Bernatsky S, Smargiassi A, Barnabe C et al. Fine particulate air pollution and systemic autoimmune rheumatic disease in two Canadian provinces. Environ Res. 2016 Apr; 146: 85-91.

40. Noonan CW, Pfau JC, Larson TC, et al. Nested case-control study of autoimmune disease in an asbestos-exposed population. Environ Health Perspect. 2006 Aug; 114(8): 1243-7.

41. Niklas K, Niklas AA, Majewski D, et al. Rheumatic diseases induced by drugs and environmental factors: the state-of-the-art - part two. Reumatologia. 2016; 54(4): 165-169.

42. Haustein UF. Scleroderma and pseudo-scleroderma: uncommon presentations. Clin Dermatol. 2005 Sep-Oct; 23(5): 480-90.

43. Saigusa R, Asano Y, Nakamura K, et al. Association of anti-RNA polymerase III antibody and silicone breast implants in patients with systemic sclerosis. J Dermatol. 2016 Jul; 43(7): 808-10. 
44. Afridi HI, Kazi TG, Brabazon D, et al. Interaction between zinc, cadmium, and lead in scalp hair samples of Pakistani and Irish smokers rheumatoid arthritis subjects in relation to controls. Biol Trace Elem Res. 2012; 148: 139-47.

45. Cooper GS, Parks CG, Treadwell EL, et al. Occupational risk factors for the development of systemic lupus erythematosus. J Rheumatol 2004; 31: 1928-33.

46. Marie I, Gehanno JF, Bubenheim M, et al. Systemic sclerosis and exposure to heavy metals: A case control study of 100 patients and 300 controls. Autoimmun Rev. 2017 Jan 27. pii: S1568-9972(17)30014-9. doi: 10.1016/j.autrev.2017.01.004.

47. Hughes M, Cooper GJ, Wilkinson J, et al. Abnormalities of selenium but not of copper homeostasis may drive tissue fibrosis in patients with systemic sclerosis. Rheumatology (Oxford). 2015 Apr; 54(4): 747-8. doi: 10.1093/rheumatology/keu470.

48. Rotondo Dottore G, Leo M, Casini G et al. Antioxidant Actions of Selenium in Orbital Fibroblasts: A Basis for the Effects of Selenium in
Graves' Orbitopathy. Thyroid. 2017 Feb; 27(2): 271-278. doi: 10.1089/ thy.2016.0397.

49. Aleksandrowicz J, Blicharski J, Dzigwska A, et al. Leuko- and oncogenesis in the light of studies on the metabolism of magnesium and its turnover in biocenosis. Acta Med Pol. 1970; 11(4): 289-302.

50. Aleksandrowicz J. Magnesium in the pathogenesis, treatment and prophylaxis of proliferating diseases of the lymphoreticular system. Przegl Lek. 1970; 26(2): 308-9.

51. Aleksandrowicz J, Gaertner L, Lechocka A, et al. Preliminary studies on magnesium and calcium levels in the serum and urine of patients with leukemia. Pol Tyg Lek. 1969 Oct 3; 24(44): 1691-3.

52. Aleksandrowicz J, Blicharski J, Dzigowska A et al. Magnesium and its role in oncogenesis. Pol Tyg Lek. 1969 Sep 9; 24(36): 1381-3.

53. Wazewska-Czyzewska M, Aleksandrowicz J, Lisiewicz J, et al. Effect of magnesium deficiency on activity of lysosomal acid phosphatase in mice lymphocytes. Patol Pol. 1977 Jan-Feb; 28(1): 39-45. 